OPEN ACCESS

Edited by:

Per-Ola Carlsson,

Uppsala University, Sweden

Reviewed by:

Vladimir Stich,

Charles University, Czechia

Tomasz Sledzinski,

Medical University of Gdańsk, Poland

*Correspondence:

Xiang Zhang

xiang.zhang02@hotmail.com

Specialty section:

This article was submitted to

Clinical and Translational Physiology,

a section of the journal

Frontiers in Physiology

Received: 06 October 2018

Accepted: 18 March 2019

Published: 02 April 2019

Citation:

Wang W, Cheng Z, Wang Y, Dai Y, Zhang X and Hu S (2019) Role of Bile

Acids in Bariatric Surgery.

Front. Physiol. 10:374

doi: 10.3389/fphys.2019.00374

\section{Role of Bile Acids in Bariatric Surgery}

\author{
Wenting Wang ${ }^{1}$, Zhiqiang Cheng ${ }^{2}$, Yanlei Wang ${ }^{2}$, Yong Dai ${ }^{2}$, Xiang Zhang ${ }^{2 \star}$ and \\ Sanyuan $\mathrm{Hu}^{2}$ \\ 'Department of Obstetrics and Gynecology, The Second Hospital of Shandong University, Jinan, China, ${ }^{2}$ Department
of General Surgery, Qilu Hospital of Shandong University, Jinan, China
}

Bariatric surgery has been proved to be effective and sustainable in the longterm weight-loss and remission of metabolic disorders. However, the underlying mechanisms are still far from fully elucidated. After bariatric surgery, the gastrointestinal tract is manipulated, either anatomically or functionally, leading to changed bile acid metabolism. Accumulating evidence has shown that bile acids play a role in metabolic regulation as signaling molecules other than digestive juice. And most of the metabolism-beneficial effects are mediated through nuclear receptor FXR and membrane receptor TGR5, as well as reciprocal influence on gut microbiota. Bile diversion procedure is also performed on animals to recapitulate the benefits of bariatric surgery. It appears that bile acid alteration is an important component of bariatric surgery, and represents a promising target for the management of metabolic disorders.

Keywords: bariatric surgery, bile acids, metabolic disorders, diabetes type 2, gut microbiota

\section{INTRODUCTION}

Bariatric surgery has been proved to be both effective and sustainable in the long-term weightloss and remission of metabolic disorders (Adams et al., 2017; Schauer et al., 2017). However, the underlying mechanisms are still far from fully elucidated. One interesting phenomenon observed after bariatric surgery is the increase of serum total bile acids. Actually, apart from the purely restrictive procedure, adjustable gastric banding (AGB) (Kohli et al., 2013a), it has been reported that all other types of bariatric surgery are associated with postsurgical increase of serum total bile acids (Steinert et al., 2013; Albaugh et al., 2015; Ferrannini et al., 2015; Han et al., 2015). And this phenomenon has raised concern about the relationships between bile acids and bariatric surgery. Bile acids are known as the "intestinal soap," for their physiological effect on facilitating dietary fat and fat-soluble vitamin absorption. It is not until the late 1990s that bile acids had been found taking effect as signaling molecules (Makishima et al., 1999). Most of the metabolic benefits of bile acids are mediated through nuclear or membrane receptors, or interaction with gut microbiota. In the present review, we focus on two major questions: (i) why serum bile acids are increased after bariatric surgery? (ii) how the altered bile acid metabolism contributes to the metabolic benefits of bariatric surgery?

\section{MECHANISMS OF SERUM BILE ACID ELEVATION AFTER BARIATRIC SURGERY}

Bile acids are synthesized from cholesterols in the liver, stored in the gallbladder and expelled into the duodenum in response to enteral stimuli. Approximately, $95 \%$ of luminal bile acids are recycled 
into the portal vein in the terminal ileum and reutilized by the liver, forming the "enterohepatic circulation." Serum bile acids represent the excess bile acids that cannot be recycled by the liver during the enterohepatic circulation and thus present in the peripheral circulation. Increased serum bile acids may derive from increased synthesis in the liver, more reabsorption in the ileum and/or decreased excretion in the feces.

In Roux-en-Y gastric bypass (RYGB) and biliopancreatic diversion (BPD), shortened route of the enterohepatic circulation (the biliopancreatic limb and the common limb) expedites the contact of luminal bile acids with the ileum, a major area for bile acid reabsorption, leading to earlier and more active bile acid reabsorption (Ferrannini et al., 2015). This explanation is also supported by an experimental procedure performed on rats termed ileal interposition (IT), in which the interposition of a segment of the ileum into the proximal jejunum shows greater bile acid reabsorption and increased serum bile acids (Cummings et al., 2013). It has been speculated that the length ratio of the biliopancreatic limb and the common limb represent a major determinant of postoperative serum bile acid concentrations. Postoperative serum bile acid concentrations are likely to be greater with longer biliopancreatic limb and shorter common limb, as evidenced by both animal (Han et al., 2015; Miyachi et al., 2016) and human studies (Mika et al., 2018). The precise mechanism has yet been elucidated. In BPD, the functional common limb left is less than one meter (Tacchino et al., 2003), and the increased luminal bile acid reabsorption cannot compensate for substantial fecal bile acid loss. Therefore, the increased hepatic bile acid synthesis also contributes to increased serum bile acids as a complementary mechanism (Scopinaro, 2006; Ferrannini et al., 2015). Compared to BPD, RYGB as well as duodenal-jejunal bypass (DJB, an experimental procedure designed to investigate the mechanism of diabetes remission with no effect on weight-loss), has enough intestinal bile acid reabsorption area, with either decreased (de Siqueira Cardinelli et al., 2019) or unchanged fecal bile acid excretion (Li et al., 2011; Bhutta et al., 2015), and the hepatic bile acid synthesis is decreased (Ferrannini et al., 2015; Zhang et al., 2016), probably due to a feedback regulation in response to the increased serum bile acids.

For vertical sleeve gastrectomy (VSG), only limited data are available regarding postsurgical serum bile acids. One human study showed immediate increase of serum bile acids after surgery (Kindel et al., 2018), while two other human studies showed unchanged serum bile acids until 1 or 2 years after surgery, with only statistically non-significant increase (Nakatani et al., 2009; Haluzikova et al., 2013). In contrast, animal studies showed consistently increased serum bile acids after VSG (Cummings et al., 2012; Myronovych et al., 2014a,b). Due to the lack of direct anatomical manipulation of the gut, the increased serum bile acids after VSG are likely to be a secondary effect of altered gastrointestinal function (Sips et al., 2018) and progressively intestinal adaption. It has been reported that VSG accelerates gastric emptying and intestinal motility (Mans et al., 2015). And Myronovych et al. (2014b) has found decreased hepatic bile acid synthesis, increased intestinal villus length and enlarged bile acid reabsorption intestinal area, which might explain the increased serum bile acids after VSG.

\section{BILE ACIDS, FXR AND BARIATRIC SURGERY}

\section{Role of FXR in Maintaining Bile Acid Homeostasis}

The farnesoid X receptor (FXR, NR1H4), an orphan member of the nuclear receptor family, was first identified as a natural receptor for bile acids in 1999 (Makishima et al., 1999; Parks et al., 1999; Wang et al., 1999). Subsequently, bile acids were found transcriptionally regulating their own synthesis and enterohepatic transport by repressing CYP7A1 (a rate-limiting enzyme for bile acid synthesis in the liver) via hepatic FXRSHP-CYP7A1 pathway (Goodwin et al., 2000; Lu et al., 2000). Later, fibroblast growth factor 19 (FGF19, in rodents ortholog FGF15), secreted mainly from the ileum in response to intestinal FXR activation, was found as an enterohepatic signal to regulate bile acid homeostasis as well (Inagaki et al., 2005). Circulating FGF15/19 within the portal venous system targets FGF receptor 4 (FGFR4) in the liver, and inhibits CYP7A1 expression and bile acid synthesis, thus working as components of a gut-liver signaling pathway that synergizes with hepatic FXR-SHP-CYP7A1 pathway to regulate bile acid homeostasis (Inagaki et al., 2005).

\section{Role of FXR in Metabolism Regulation}

The metabolism-regulating effect of FXR is more complex compared to bile acid homeostasis maintenance. A variety of genetic and pharmacological animal models have been established to investigate the role of FXR signaling in body weight management and metabolism regulation. Whole body FXR knock-out mice $\left(F X R^{-/-}\right)$displayed impaired glucose disposal and elevated blood glucose concentrations, as a result of blunted insulin pathway in the liver, peripheral adipose tissue and skeletal muscles, although FXR is not expressed in skeletal muscles (Cariou et al., 2006; Ma et al., 2006). Meantime, plasma triglycerides, cholesterols and free fatty acids were elevated, with severe liver fat accumulation, however, surprisingly, this kind of mouse model was protected from diet- or geneticallyinduced obesity, probably because the adipocyte differentiation was impaired in the absence of FXR (Sinal et al., 2000; Cariou et al., 2006). In contrast, supplementation of whole-body FXR agonist (GW4064) in ob/ob mice has multiple beneficial effects, indicating that FXR could be a potential therapeutic target for metabolic disorders and bariatric surgery (Cariou et al., 2006).

Since FXR is abundant in the liver and small intestine, some studies concentrate on its tissue-specific signaling. In the liver, FXR activation lowers plasma triglycerides via FXR-SHP(SREBP-1c) pathway (Watanabe et al., 2004), and improves hyperglycemia by inhibiting gluconeogenesis and improving insulin sensitivity (Zhang et al., 2006). In contrast, liver-specific $\mathrm{FXR}^{-/-}$mice showed increased plasma triglycerides, and unlike 
whole-body FXR knock-out, were not protected from dietinduced obesity and insulin resistance (Watanabe et al., 2004). In the small intestine, the physiological role of FXR is primarily mediated by FGF15/19 whose metabolism-beneficial functions resemble those of insulin in stimulating protein and glycogen synthesis and inhibiting gluconeogenesis, but does not lead to body weight gain or hepatic fat accumulation (Kir et al., 2011).

\section{Bile Acid-FXR Pathway in Bariatric Surgery}

Since bile acids are natural ligands for FXR, it has been believed that bile acid-FXR pathway contribute to the beneficial effects of bariatric surgery. However, no consensus has been reached so far. The essential role of FXR in bariatric surgery was first demonstrated by a milestone research published in 2014. The ability of VSG to reduce body weight and improve glucose tolerance was substantially reduced in the state of genetic disruption of FXR (Ryan et al., 2014). Nevertheless, the results were later questioned by the authors themselves, as the whole body FXR knock-out mice per se were protected from diet-induced obesity as well as glucose intolerance, and the observational window might not be long enough (Bozadjieva et al., 2018). Bariatric surgery alters bile acid drainage, either anatomically or functionally, and changes luminal bile acid concentrations and composition. Activation of intestinal FXR by bile acids increases FGF19 following bariatric surgery in humans and represents one mechanism accounting for postsurgical metabolic improvement (Jansen et al., 2011; Pournaras et al., 2012; Gerhard et al., 2013; Haluzikova et al., 2013; Jorgensen et al., 2015; Sachdev et al., 2016). One human study did not support the contribution of FGF19 to the benefit of RYGB, as the increase of FGF19 did not parallel the improvement of glucose tolerance (Jorgensen et al., 2015). And animal studies have shown that both intestine-specific FXR agonist (Fexaramine) (Fang et al., 2015) and antagonist (Glycine-muricholic acid) (Jiang et al., 2015) have the capacity of reducing body weight and improving metabolism. Interestingly, alteration of luminal bile acids after bariatric surgery did not guarantee activation of intestinal FXR. On the contrary, intestinal FXR was intact or even repressed (Kohli et al., 2013b; Goncalves et al., 2015). These unexpected phenomena have been thought closely related to the intestinal bile acid milieu, as the physiological effect of each individual bile acid differs in interacting with FXR, with chenodeoxycholic acid (CDCA) being a potent agonist, deoxycholic acid (DCA) being a moderate agonist (Parks et al., 1999), yet ursodeoxycholic acid (UDCA) (Mueller et al., 2015), and muricholic acid (MCA, only in rodents) being antagonists (Jiang et al., 2015). Surprisingly, intestinal FXR repression also leads to metabolic benefits. One study reported that FXR repression within intestinal $\mathrm{L}$ cells led to glucagon-like peptide 1 (GLP-1) secretion hence improving glucose metabolism (Trabelsi et al., 2015). This finding may also partly explain the paradox between intestinal bile supplementation and bile deprivation (e.g., bile sequestrant), both of which have been confirmed effective in enhancing GLP-1 secretion (Trabelsi et al., 2015; Zhang et al., 2016).

It should be noted that most of the studies identifying intestinal FXR repression as a positive metabolic regulator are from animal models, suggesting that the gut may respond differently to FXR-specific targeting between animals and humans.

\section{BILE ACIDS, TGR5, AND BARIATRIC SURGERY}

G protein coupled receptor 1, also known as TGR5 (GPR131), was discovered as a membrane-type receptor for bile acids in 2002 (Maruyama et al., 2002) and has been confirmed as another essential receptor in maintaining glucose homeostasis after sleeve gastrectomy (McGavigan et al., 2017). However, one latest report revealed that the metabolic benefits were still maintained in $\mathrm{Tgr5}^{-/-}$, but not $\mathrm{Fxr}^{\Delta} / E$ mice after a bariatric procedure, suggesting a less important role for TGR5 as opposed to intestinal FXR (Albaugh et al., 2018). Unlike FXR, each individual bile acid is capable of activating TGR5, only with different potency (conjugated form > unconjugated form, LCA > DCA > CDCA > CA) (Maruyama et al., 2002). Therefore, after bariatric surgery, the increased luminal or serum bile acids are able to elicit TGR5-mediated metabolic benefits, and appear to make a complementary contribution to the metabolic benefits of bariatric surgery.

\section{Luminal Bile Acids, TGR5, and Incretins}

In the distal small intestine, TGR5 activation within enteroendocrine $\mathrm{L}$ cells in response to luminal bile acids have been confirmed as a critical signaling pathway in promoting GLP-1 secretion in both animal models (Thomas et al., 2009) and humans (Wu et al., 2013). Following bariatric surgery, as a result of gut manipulation and increased luminal bile acids, serum GLP-1 has been found ubiquitously elevated, either acutely (le Roux et al., 2007) or gradually (Borg et al., 2006), and is now referred to as an important, albeit not exclusive, mechanism of bariatric surgery in improving glucose tolerance (Madsbad and Holst, 2014). GLP-1 and glucose-dependent insulinotropic polypeptide (GIP) are known as "incretin hormones" as they mediate the incretin effect, defined as the increased stimulation of insulin secretion elicited by oral compared with intravenous administration of glucose under similar plasma glucose levels (Wu et al., 2016). In health, the incretin effect accounts for $70 \%$ of postprandial insulin secretion after oral ingestion of glucose in a glucosedependent fashion (Nauck et al., 1986). In T2DM, the incretin effect is markedly impaired as a result of the loss of insulinotropic effect of GIP, while GLP-1 still maintains its pleiotropic physiological functions including stimulating insulin secretion, improving hepatic insulin sensitivity and suppressing appetite (Wu et al., 2014, 2016), which might account for the partially restored incretin effect after bariatric surgery (Laferrere et al., 2007). A latest "proof of concept" study 
has proved that the distal, as opposed to proximal, small intestine is superior in modulating postprandial glucose metabolism in both health and T2DM, of which GLP-1 plays a major role (Zhang et al., 2019). Currently, GLP-1 analogs as well as dipeptidyl peptidase-4 (DDP4, an enzyme for degradation of incretins) inhibitors have been commercialized for the management of T2DM.

\section{Serum Bile Acids, TGR5, and Energy Expenditure}

In the skeletal muscle and brown adipose, increased serum bile acids have the capacity of converting tetraiodothyronine (T4) to triiodothyronine (T3) through TGR5, leading to more energy expenditure, which might facilitate weight loss (Kohli et al., 2013a; Broeders et al., 2015; Watanabe et al., 2006). However, the relevance between TGR5-mediated energy expenditure and bariatric surgery has not been clarified. In a human study, increased skeletal gene expressions of TGR5 downstream targets (mitochondrial COX IV and Kir6.2) were detected after RYGB, but no correlation with resting energy expenditure was found (Kohli et al., 2013a). In addition, the majority of studies suggest decreased energy expenditure after bariatric surgery, which might be a secondary effect of weight loss (Benedetti et al., 2000; Carrasco et al., 2007). In contrast, increased weight-adjusted energy expenditure was also present in some studies (Faria et al., 2012; Rabl et al., 2014). As energy metabolism is related to many factors, such as body composition, exercise or hormones, whether TGR5-mediated energy regulation plays a role in weight-loss after bariatric surgery still warrants further research.

\section{MICROBIOTA AND BILE ACIDS}

\section{Role of Gut Microbiota in Metabolic Disorders}

Gut microbiota, also named as "The Second Genome," consists of more than $10^{14}$ microorganisms, most of which have not been fully investigated (Turnbaugh et al., 2007). Five phyla dominate human gut community, including Actinobacteria, Bacteroidetes, Firmicutes, Proteobacteria, and Verrucomicrobia, of which Bacteroidetes and Firmicutes account for over 90\% of the whole gut microbiota (Tilg and Kaser, 2011). Along the whole gut, microbiota is not uniformly distributed. Both the abundance and diversity are increased from the proximal to distal gut (Prakash et al., 2011). In the stomach, only bacteria resistant to acidic environment survive (e.g., Lactobacillus or Streptococcus or Veillonella) (Dicksved et al., 2009), while in the large intestine, where the intrinsic environment is suitable for bacterial growth, lives most of the gut bacteria (Prakash et al., 2011). Gut microbiota begins to colonize since newborns, and is related to certain pathological states in adulthood. An identical twin study showed that different physiologic states (obese versus lean) are associated with distinct gut microbiota, despite the same genetic background
(Turnbaugh et al., 2009). In obesity, the majority of animal and human studies have demonstrated that the diversity of gut microbiota is decreased, and Phylum Firmicutes is increased at the expense of Phylum Bacteroidetes, independent of food consumption (Ley et al., 2006; Turnbaugh et al., 2006, 2009). In T2DM, inconsistent results have been reported. Larsen et al. found that the proportions of Phylum Firmicutes and Class Clostridia were significantly reduced in T2DM, and Bacteroidetes/Firmicutes ratio was correlated positively and significantly with plasma glucose concentrations (Larsen et al., 2010). Qin et al. used a deep shotgun sequencing method analyzing gut microbial DNA from 345 Chinese individuals, and found that type 2 patients had a moderate degree of gut bacterial dysbiosis, a decline in butyrateproducing bacteria and an increase in diverse opportunistic pathological bacteria (Qin et al., 2012). They also pointed out that compared to inflammatory bowel disease (IBD), the degree of gut microbiota changes in T2DM was not that substantial. Since gut microbiota is influenced by various factors such as life style, dietary, disease, medication, and surgery, the currently available results should be interpreted with caution, and may not be used as a marker for T2DM monitoring at the moment.

\section{Changes of Gut Microbiota After Bariatric Surgery}

Bile acids and gut microbiota are reciprocally affected and are both altered by bariatric surgery. Within the enterohepatic circulation, bile acids in the distal ileum and colon undergo hydrolysis (deconjugation) and subsequent transformation into secondary bile acids. These processes require the catalysis of bile salt hydrolases (BSHs, produced by certain gut bacteria) and the bacterial activity of $7 \alpha$-dehydroxylation (Ridlon et al., 2006). Meantime, both gut bile acid concentrations and compositions have direct influence on gut microbiota. Increased luminal bile acid concentrations have anti-bacterial effects, killing certain strains of bacterium and make some other bacteria (e.g., Bilophila wadsworthia) thrive (Noh and Gilliland, 1993; Devkota et al., 2012). Furthermore, some individual bile acids are capable of activating enteral FXR, thus leading to enteroprotection by promoting related gene expressions and inhibits bacterial overgrowth and mucosal injury in the ileum. All these effects indicate the existence of indirect influence on gut microbiota by gut bile acids (Inagaki et al., 2006). Following bariatric surgery, gut microbiota is changed. In RYGB, at the phylum level, Proteobacteria has been reported to be increased at the expense of Firmicutes (Zhang et al., 2009; Tremaroli et al., 2015), while Bacteroidetes has been found inconsistently changed, either increased (Furet et al., 2010) or decreased (Li et al., 2011; Graessler et al., 2013). In VSG, both animal and human studies showed increased Bacteroidetes and decreased Firmicutes, and rebuilt of the gut microbiota diversity (Jahansouz et al., 2017; Murphy et al., 2017; Shao et al., 2018). At the species level, the outcomes have been inconsistently reported across different studies, further increasing the difficulty of gut microbiota research. 


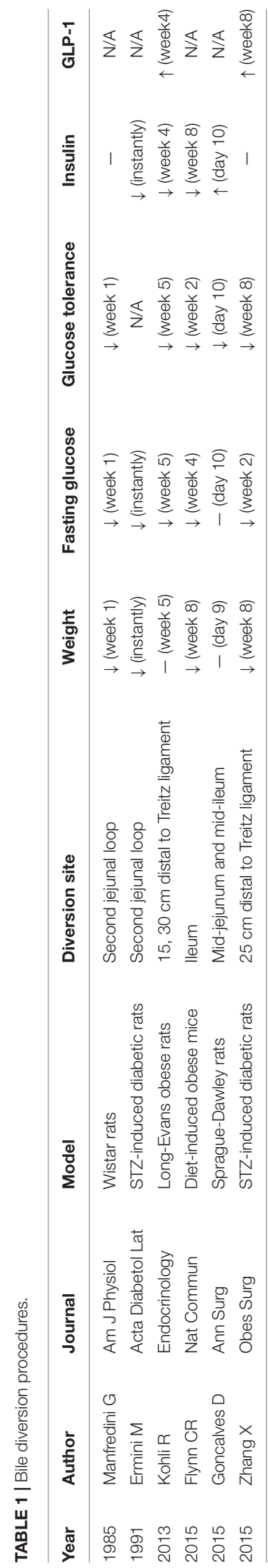

As to the interaction of bile acids and gut microbiota after bariatric surgery, it's similar to an old question: "which came first, the chicken or the egg?" We do not have direct and strong evidence at the moment, but assume that the instantly altered luminal bile acids following bariatric surgery might be the driving force, and the final phenotype of luminal bile acids and gut microbiota is a result of gradual interaction and adaption of each other.

\section{Gut Microbiota After Bariatric Surgery: A Signal Carrier?}

Another question to be answered is how gut microbiota affects the host after bariatric surgery. In fact, the contribution of gut microbiota to the metabolic benefits after bariatric surgery has not been clarified. Based on currently available reports, gut microbiota is likely to be a signal carrier and transmits signals that are able to play a role even in a different host. Direct evidence comes from fecal microbiota transplantation (FMT). Patients with metabolic syndrome experienced significantly increased insulin sensitivity after receiving gut microbiota from lean donors (Vrieze et al., 2012). However, the improvement of insulin sensitivity disappeared at 12 weeks after FMT, suggesting that the beneficial effect of FMT might not last in absence of sustainable exogenous stimulations. Metabolic benefits were also observed in FMT from bariatric surgery recipient donors to mice (Tremaroli et al., 2015), but we do not know whether the effect of bariatric surgery could be recapitulated by FMT in human recipient as well.

Due to technical reasons, it's difficult to observe the dynamic change of luminal bile acids and gut microbiota frequently after bariatric surgery. And a Dutch population-based cohort showed that the influence of currently realized exogenous and intrinsic host factors could only explain $18.7 \%$ of the inter-individual variation of microbial composition (Zhernakova et al., 2016). Therefore, the knowledge of gut microbiota is far from enough and warrants future research.

\section{BILE DIVERSION PROCEDURES}

In order to isolate the contribution of bile acids to the metabolic benefits of bariatric surgery, some studies have tried to manipulate bile flow directly without altering the gastrointestinal tract (Table 1). Bile diversion (BD) procedure in rats was performed as early as the 1980s, even before the discovery of GLP-1, to investigate the influence of rerouting of bile flow on glucose homeostasis. This early study showed $10 \%$ less weight of rats undergoing BD than control, decreased fasting blood glucose and improved glucose tolerance, with no effect on insulin secretion (Manfredini et al., 1985). Another study in 1991 using streptozocin (STZ) -induced diabetic rats showed similar results, except an immediate return of insulin to normal level after surgery (Ermini et al., 1991). It is not until 2013 that the $\mathrm{BD}$ procedure had regained attention. Subsequent studies have demonstrated comparable effects of $\mathrm{BD}$ with gastric bypass procedure in glucose control 
in normal rats (Goncalves et al., 2015), diet-induced obese rats (Kohli et al., 2013b), and mice (Flynn et al., 2015). However, under diabetic state, the effects of BD were attenuated compared to DJB, with limited glucose-lowering effect observed. Therefore, to our knowledge, bile acid manipulation appears to be capable of recapitulating the effects of bariatric surgery only in nondiabetic state.

\section{CONCLUSION}

Bile acids play a role in metabolic regulation through various pathways. Alteration of bile acid metabolism is an important component of bariatric surgery, and represents a promising target for the management of metabolic disorders.

\section{REFERENCES}

Adams, T. D., Davidson, L. E., Litwin, S. E., Kim, J., Kolotkin, R. L., Nanjee, M. N., et al. (2017). Weight and metabolic outcomes 12 years after gastric bypass. New Engl. J. Med. 377, 1143-1155. doi: 10.1056/NEJMoa1700459

Albaugh, V. L., Banan, B., Antoun, J., Xiong, Y., Guo, Y., Ping, J., et al. (2018). Role of bile acids and GLP-1 in mediating the metabolic improvements of bariatric surgery. Gastroenterology 156, 1041-1051.e4. doi: 10.1053/j.gastro.2018.11.017

Albaugh, V. L., Flynn, C. R., Cai, S., Xiao, Y., Tamboli, R. A., and Abumrad, N. N. (2015). Early increases in bile acids post roux-en-Y gastric bypass are driven by insulin-sensitizing, secondary bile acids. J. Clin. Endocrinol. Metab. 100, E1225-E1233. doi: 10.1210/jc.2015-2467

Benedetti, G., Mingrone, G., Marcoccia, S., Benedetti, M., Giancaterini, A., Greco, A. V., et al. (2000). Body composition and energy expenditure after weight loss following bariatric surgery. J. Am. Coll. Nutr. 19, 270-274. doi: 10.1080/ 07315724.2000 .10718926

Bhutta, H. Y., Rajpal, N., White, W., Freudenberg, J. M., Liu, Y., Way, J., et al. (2015). Effect of Roux-en-Y gastric bypass surgery on bile acid metabolism in normal and obese diabetic rats. PLoS One 10:e0122273. doi: 10.1371/journal. pone. 0122273

Borg, C. M., le Roux, C. W., Ghatei, M. A., Bloom, S. R., Patel, A. G., and Aylwin, S. J. (2006). Progressive rise in gut hormone levels after Roux-en-Y gastric bypass suggests gut adaptation and explains altered satiety. Br. J. Surg. 93, 210-215. doi: 10.1002/bjs.5227

Bozadjieva, N., Heppner, K. M., and Seeley, R. J. (2018). Targeting FXR and FGF19 to treat metabolic diseases-lessons learned from bariatric surgery. Diabetes 67, 1720-1728. doi: 10.2337/dbi17-0007

Broeders, E. P., Nascimento, E. B., Havekes, B., Brans, B., Roumans, K. H., Tailleux, A., et al. (2015). The bile acid chenodeoxycholic acid increases human brown adipose tissue activity. Cell Metab. 22, 418-426. doi: 10.1016/j.cmet.2015. 07.002

Cariou, B., van Harmelen, K., Duran-Sandoval, D., van Dijk, T. H., Grefhorst, A., Abdelkarim, M., et al. (2006). The farnesoid X receptor modulates adiposity and peripheral insulin sensitivity in mice. J. Biol. Chem. 281, 11039-11049. doi: 10.1074/jbc.M510258200

Carrasco, F., Papapietro, K., Csendes, A., Salazar, G., Echenique, C., Lisboa, C., et al. (2007). Changes in resting energy expenditure and body composition after weight loss following Roux-en-Y gastric bypass. Obes. Surg. 17, 608-616. doi: 10.1007/s11695-007-9117-z

Cummings, B. P., Bettaieb, A., Graham, J. L., Kim, J., Ma, F., Shibata, N., et al. (2013). Bile-acid-mediated decrease in endoplasmic reticulum stress: a potential contributor to the metabolic benefits of ileal interposition surgery in UCDT2DM rats. Dis. Model. Mech. 6, 443-456. doi: 10.1242/dmm.010421

Cummings, B. P., Bettaieb, A., Graham, J. L., Stanhope, K. L., Kowala, M., Haj, F. G., et al. (2012). Vertical sleeve gastrectomy improves glucose and lipid metabolism and delays diabetes onset in UCD-T2DM rats. Endocrinology 153, 3620-3632. doi: 10.1210/en.2012-1131

\section{AUTHOR CONTRIBUTIONS}

WW was involved in literature searching and writing of the manuscript. ZC, YW, YD, XZ, and $\mathrm{SH}$ were involved in conception and assist in writing. XZ was involved in conception, design, and coordination of the work. All authors critically reviewed the manuscript and have approved the publication of this final version of the manuscript. $\mathrm{XZ}$ and $\mathrm{SH}$ are the guarantors of this work.

\section{FUNDING}

The present work has been supported by National Natural Science Foundation of China (Project ID: 81700708/H0712).

de Siqueira Cardinelli, C., Torrinhas, R. S., Sala, P., Pudenzi, M. A., Fernando, F. A. C., Marques da Silva, M., et al. (2019). Fecal bile acid profile after Rouxen-Y gastric bypass and its association with the remission of type 2 diabetes in obese women: a preliminary study. Clin. Nutr. doi: 10.1016/j.clnu.2018.12.028 [Epub ahead of print].

Devkota, S., Wang, Y., Musch, M. W., Leone, V., Fehlner-Peach, H., Nadimpalli, A., et al. (2012). Dietary-fat-induced taurocholic acid promotes pathobiont expansion and colitis in Il10-/- mice. Nature 487, 104-108. doi: 10.1038/ nature 11225

Dicksved, J., Lindberg, M., Rosenquist, M., Enroth, H., Jansson, J. K., and Engstrand, L. (2009). Molecular characterization of the stomach microbiota in patients with gastric cancer and in controls. J. Med. Microbiol. 58(Pt 4), 509-516. doi: 10.1099/jmm.0.007302-0

Ermini, M., Iaconis, E., and Mori, A. (1991). The effects of bilio-jejunal diversion on streptozotocin diabetes in the rat. Acta Diabetol. Lat. 28, 79-89. doi: 10.1007/ BF02732117

Fang, S., Suh, J. M., Reilly, S. M., Yu, E., Osborn, O., Lackey, D., et al. (2015). Intestinal FXR agonism promotes adipose tissue browning and reduces obesity and insulin resistance. Nat. Med. 21, 159-165. doi: 10.1038/nm.3760

Faria, S. L., Faria, O. P., Buffington, C., de Almeida Cardeal, M., and Rodrigues de Gouvea, H. (2012). Energy expenditure before and after Roux-en-Y gastric bypass. Obes. Surg. 22, 1450-1455. doi: 10.1007/s11695-012-0672-6

Ferrannini, E., Camastra, S., Astiarraga, B., Nannipieri, M., Castro-Perez, J., Xie, D., et al. (2015). Increased bile acid synthesis and deconjugation after biliopancreatic diversion. Diabetes 64, 3377-3385. doi: 10.2337/db15-0214

Flynn, C. R., Albaugh, V. L., Cai, S., Cheung-Flynn, J., Williams, P. E., Brucker, R. M., et al. (2015). Bile diversion to the distal small intestine has comparable metabolic benefits to bariatric surgery. Nat. Commun. 6:7715. doi: 10.1038/ ncomms 8715

Furet, J. P., Kong, L. C., Tap, J., Poitou, C., Basdevant, A., Bouillot, J. L., et al. (2010). Differential adaptation of human gut microbiota to bariatric surgery-induced weight loss: links with metabolic and low-grade inflammation markers. Diabetes 59, 3049-3057. doi: 10.2337/db10-0253

Gerhard, G. S., Styer, A. M., Wood, G. C., Roesch, S. L., Petrick, A. T., Gabrielsen, J., et al. (2013). A role for fibroblast growth factor 19 and bile acids in diabetes remission after Roux-en-Y gastric bypass. Diabetes Care 36, 1859-1864. doi: $10.2337 / \mathrm{dc} 12-2255$

Goncalves, D., Barataud, A., De Vadder, F., Vinera, J., Zitoun, C., Duchampt, A., et al. (2015). Bile routing modification reproduces key features of gastric bypass in rat. Ann. Surg. 262, 1006-1015. doi: 10.1097/SLA.0000000000001121

Goodwin, B., Jones, S. A., Price, R. R., Watson, M. A., McKee, D. D., Moore, L. B., et al. (2000). A regulatory cascade of the nuclear receptors FXR, SHP-1, and LRH-1 represses bile acid biosynthesis. Mol. Cell 6, 517-526. doi: 10.1016/ S1097-2765(00)00051-4

Graessler, J., Qin, Y., Zhong, H., Zhang, J., Licinio, J., Wong, M. L., et al. (2013). Metagenomic sequencing of the human gut microbiome before and after bariatric surgery in obese patients with type 2 diabetes: correlation with 
inflammatory and metabolic parameters. Pharmacogenomics $J$ 13, 514-522. doi: $10.1038 /$ tpj. 2012.43

Haluzikova, D., Lacinova, Z., Kavalkova, P., Drapalova, J., Krizova, J., Bartlova, M., et al. (2013). Laparoscopic sleeve gastrectomy differentially affects serum concentrations of FGF-19 and FGF-21 in morbidly obese subjects. Obesity 21, 1335-1342. doi: 10.1002/oby.20208

Han, H., Wang, L., Du, H., Jiang, J., Hu, C., Zhang, G., et al. (2015). Expedited biliopancreatic juice flow to the distal gut benefits the diabetes control after duodenal-jejunal bypass. Obes. Surg. 25, 1802-1809. doi: 10.1007/s11695-0151633-7

Inagaki, T., Choi, M., Moschetta, A., Peng, L., Cummins, C. L., McDonald, J. G., et al. (2005). Fibroblast growth factor 15 functions as an enterohepatic signal to regulate bile acid homeostasis. Cell Metab. 2, 217-225. doi: 10.1016/j.cmet.2005. 09.001

Inagaki, T., Moschetta, A., Lee, Y. K., Peng, L., Zhao, G., Downes, M., et al. (2006). Regulation of antibacterial defense in the small intestine by the nuclear bile acid receptor. Proc. Natl. Acad. Sci. U.S.A. 103, 3920-3925. doi: 10.1073/pnas. 0509592103

Jahansouz, C., Staley, C., Bernlohr, D. A., Sadowsky, M. J., Khoruts, A., and Ikramuddin, S. (2017). Sleeve gastrectomy drives persistent shifts in the gut microbiome. Surg. Obes. Relat. Dis. 13, 916-924. doi: 10.1016/j.soard.2017.01. 003

Jansen, P. L., van Werven, J., Aarts, E., Berends, F., Janssen, I., Stoker, J., et al. (2011). Alterations of hormonally active fibroblast growth factors after Rouxen-Y gastric bypass surgery. Dig. Dis. 29, 48-51. doi: 10.1159/000324128

Jiang, C., Xie, C., Lv, Y., Li, J., Krausz, K. W., Shi, J., et al. (2015). Intestineselective farnesoid X receptor inhibition improves obesity-related metabolic dysfunction. Nat. Commun. 6:10166. doi: 10.1038/ncomms10166

Jorgensen, N. B., Dirksen, C., Bojsen-Moller, K. N., Kristiansen, V. B., Wulff, B. S., Rainteau, D., et al. (2015). Improvements in glucose metabolism early after gastric bypass surgery are not explained by increases in total bile acids and fibroblast growth factor 19 concentrations. J. Clin. Endocrinol. Metab. 100, E396-E406. doi: 10.1210/jc.2014-1658

Kindel, T. L., Krause, C., Helm, M. C., McBride, C. L., Oleynikov, D., Thakare, R., et al. (2018). Increased glycine-amidated hyocholic acid correlates to improved early weight loss after sleeve gastrectomy. Surg. Endosc. 32, 805-812. doi: 10. 1007/s00464-017-5747-y

Kir, S., Beddow, S. A., Samuel, V. T., Miller, P., Previs, S. F., Suino-Powell, K., et al. (2011). FGF19 as a postprandial, insulin-independent activator of hepatic protein and glycogen synthesis. Science 331, 1621-1624. doi: 10.1126/science. 1198363

Kohli, R., Bradley, D., Setchell, K. D., Eagon, J. C., Abumrad, N., and Klein, S. (2013a). Weight loss induced by Roux-en-Y gastric bypass but not laparoscopic adjustable gastric banding increases circulating bile acids. J. Clin. Endocrinol. Metab. 98, E708-E712. doi: 10.1210/jc.20123736

Kohli, R., Setchell, K. D., Kirby, M., Myronovych, A., Ryan, K. K., Ibrahim, S. H., et al. (2013b). A surgical model in male obese rats uncovers protective effects of bile acids post-bariatric surgery. Endocrinology 154, 2341-2351. doi: 10.1210/ en.2012-2069

Laferrere, B., Heshka, S., Wang, K., Khan, Y., McGinty, J., Teixeira, J., et al. (2007). Incretin levels and effect are markedly enhanced 1 month after Roux-en-Y gastric bypass surgery in obese patients with type 2 diabetes. Diabetes Care 30, 1709-1716. doi: $10.2337 / \mathrm{dc} 06-1549$

Larsen, N., Vogensen, F. K., van den Berg, F. W., Nielsen, D. S., Andreasen, A. S., Pedersen, B. K., et al. (2010). Gut microbiota in human adults with type 2 diabetes differs from non-diabetic adults. PLoS One 5:e9085. doi: 10.1371/ journal.pone.0009085

le Roux, C. W., Welbourn, R., Werling, M., Osborne, A., Kokkinos, A., Laurenius, A., et al. (2007). Gut hormones as mediators of appetite and weight loss after Roux-en-Y gastric bypass. Ann. Surg. 246, 780-785. doi: 10.1097/SLA. 0b013e3180caa3e3

Ley, R. E., Turnbaugh, P. J., Klein, S., and Gordon, J. I. (2006). Microbial ecology: human gut microbes associated with obesity. Nature 444, 1022-1023. doi: $10.1038 / 4441022$ a

Li, J. V., Ashrafian, H., Bueter, M., Kinross, J., Sands, C., le Roux, C. W., et al. (2011). Metabolic surgery profoundly influences gut microbial-host metabolic cross-talk. Gut 60, 1214-1223. doi: 10.1136/gut.2010.234708
Lu, T. T., Makishima, M., Repa, J. J., Schoonjans, K., Kerr, T. A., Auwerx, J., et al. (2000). Molecular basis for feedback regulation of bile acid synthesis by nuclear receptors. Mol. Cell 6, 507-515. doi: 10.1016/S1097-2765(00)00050-2

Ma, K., Saha, P. K., Chan, L., and Moore, D. D. (2006). Farnesoid X receptor is essential for normal glucose homeostasis. J. Clin. Invest. 116, 1102-1109. doi: 10.1172/JCI25604

Madsbad, S., and Holst, J. J. (2014). GLP-1 as a mediator in the remission of type 2 diabetes after gastric bypass and sleeve gastrectomy surgery. Diabetes 63 , 3172-3174. doi: $10.2337 / \mathrm{db} 14-0935$

Makishima, M., Okamoto, A. Y., Repa, J. J., Tu, H., Learned, R. M., Luk, A., et al. (1999). Identification of a nuclear receptor for bile acids. Science 284, 1362-1365. doi: 10.1126/science.284.5418.1362

Manfredini, G., Ermini, M., Scopsi, L., Bonaguidi, F., and Ferrannini, E. (1985). Internal biliary diversion improves glucose tolerance in the rat. Am. J. Physiol. 249(4 Pt 1), G519-G527. doi: 10.1152/ajpgi.1985.249.4.G519

Mans, E., Serra-Prat, M., Palomera, E., Sunol, X., and Clave, P. (2015). Sleeve gastrectomy effects on hunger, satiation, and gastrointestinal hormone and motility responses after a liquid meal test. Am. J. Clin. Nutr. 102, 540-547. doi: 10.3945/ajcn.114.104307

Maruyama, T., Miyamoto, Y., Nakamura, T., Tamai, Y., Okada, H., Sugiyama, E., et al. (2002). Identification of membrane-type receptor for bile acids (M-BAR). Biochem. Biophys. Res. Commun. 298, 714-719. doi: 10.1016/S0006-291X(02) 02550-0

McGavigan, A. K., Garibay, D., Henseler, Z. M., Chen, J., Bettaieb, A., Haj, F. G., et al. (2017). TGR5 contributes to glucoregulatory improvements after vertical sleeve gastrectomy in mice. Gut 66, 226-234. doi: 10.1136/gutjnl-2015-309871

Mika, A., Kaska, L., Proczko-Stepaniak, M., Chomiczewska, A., Swierczynski, J., Smolenski, R. T., et al. (2018). Evidence that the length of bile loop determines serum bile acid concentration and glycemic control after bariatric surgery. Obes. Surg. 28, 3405-3414. doi: 10.1007/s11695-018-3314-9

Miyachi, T., Nagao, M., Shibata, C., Kitahara, Y., Tanaka, N., Watanabe, K., et al. (2016). Biliopancreatic limb plays an important role in metabolic improvement after duodenal-jejunal bypass in a rat model of diabetes. Surgery 159, 1360-1371. doi: 10.1016/j.surg.2015.11.027

Mueller, M., Thorell, A., Claudel, T., Jha, P., Koefeler, H., Lackner, C., et al. (2015). Ursodeoxycholic acid exerts farnesoid X receptor-antagonistic effects on bile acid and lipid metabolism in morbid obesity. J. Hepatol. 62, 1398-1404. doi: 10.1016/j.jhep.2014.12.034

Murphy, R., Tsai, P., Jullig, M., Liu, A., Plank, L., and Booth, M. (2017). Differential changes in gut microbiota after gastric bypass and sleeve gastrectomy bariatric surgery vary according to diabetes remission. Obes. Surg. 27, 917-925. doi: 10.1007/s11695-016-2399-2

Myronovych, A., Kirby, M., Ryan, K. K., Zhang, W., Jha, P., Setchell, K. D., et al. (2014a). Vertical sleeve gastrectomy reduces hepatic steatosis while increasing serum bile acids in a weight-loss-independent manner. Obesity 22, 390-400. doi: 10.1002/oby.20548

Myronovych, A., Salazar-Gonzalez, R. M., Ryan, K. K., Miles, L., Zhang, W., Jha, P., et al. (2014b). The role of small heterodimer partner in nonalcoholic fatty liver disease improvement after sleeve gastrectomy in mice. Obesity 22, 2301-2311. doi: 10.1002/oby.20890

Nakatani, H., Kasama, K., Oshiro, T., Watanabe, M., Hirose, H., and Itoh, H. (2009). Serum bile acid along with plasma incretins and serum high-molecular weight adiponectin levels are increased after bariatric surgery. Metabolism 58, 1400-1407. doi: 10.1016/j.metabol.2009.05.006

Nauck, M. A., Homberger, E., Siegel, E. G., Allen, R. C., Eaton, R. P., Ebert, R., et al. (1986). Incretin effects of increasing glucose loads in man calculated from venous insulin and C-peptide responses. J. Clin. Endocrinol. Metab. 63, 492-498. doi: 10.1210/jcem-63-2-492

Noh, D. O., and Gilliland, S. E. (1993). Influence of bile on cellular integrity and beta-galactosidase activity of Lactobacillus acidophilus. J. Dairy Sci. 76, 1253-1259. doi: 10.3168/jds.S0022-0302(93)77454-8

Parks, D. J., Blanchard, S. G., Bledsoe, R. K., Chandra, G., Consler, T. G., Kliewer, S. A., et al. (1999). Bile acids: natural ligands for an orphan nuclear receptor. Science 284, 1365-1368. doi: 10.1126/science.284.5418.1365

Pournaras, D. J., Glicksman, C., Vincent, R. P., Kuganolipava, S., AlaghbandZadeh, J., Mahon, D., et al. (2012). The role of bile after Roux-en-Y gastric bypass in promoting weight loss and improving glycaemic control. Endocrinology 153, 3613-3619. doi: 10.1210/en.2011-2145 
Prakash, S., Tomaro-Duchesneau, C., Saha, S., and Cantor, A. (2011). The gut microbiota and human health with an emphasis on the use of microencapsulated bacterial cells. J. Biomed. Biotechnol. 2011:981214. doi: $10.1155 / 2011 / 981214$

Qin, J., Li, Y., Cai, Z., Li, S., Zhu, J., Zhang, F., et al. (2012). A metagenomewide association study of gut microbiota in type 2 diabetes. Nature 490, 55-60. doi: 10.1038/nature11450

Rabl, C., Rao, M. N., Schwarz, J. M., Mulligan, K., and Campos, G. M. (2014). Thermogenic changes after gastric bypass, adjustable gastric banding or diet alone. Surgery 156, 806-812. doi: 10.1016/j.surg.2014.06.070

Ridlon, J. M., Kang, D. J., and Hylemon, P. B. (2006). Bile salt biotransformations by human intestinal bacteria. J. Lipid Res. 47, 241-259. doi: 10.1194/jlr. R500013-JLR200

Ryan, K. K., Tremaroli, V., Clemmensen, C., Kovatcheva-Datchary, P., Myronovych, A., Karns, R., et al. (2014). FXR is a molecular target for the effects of vertical sleeve gastrectomy. Nature 509, 183-188. doi: 10.1038/nature13135

Sachdev, S., Wang, Q., Billington, C., Connett, J., Ahmed, L., Inabnet, W., et al. (2016). FGF 19 and bile acids increase following Roux-en-Y gastric bypass but not after medical management in patients with type 2 diabetes. Obes. Surg. 26, 957-965. doi: 10.1007/s11695-015-1834-0

Schauer, P. R., Bhatt, D. L., Kirwan, J. P., Wolski, K., Aminian, A., Brethauer, S. A., et al. (2017). Bariatric surgery versus intensive medical therapy for diabetes - 5year outcomes. New Engl. J. Med. 376, 641-651. doi: 10.1056/NEJMoa1600869

Scopinaro, N. (2006). Biliopancreatic diversion: mechanisms of action and longterm results. Obes. Surg. 16, 683-689. doi: 10.1381/096089206777346637

Shao, Y., Shen, Q., Hua, R., Evers, S. S., He, K., and Yao, Q. (2018). Effects of sleeve gastrectomy on the composition and diurnal oscillation of gut microbiota related to the metabolic improvements. Surg. Obes. Relat. Dis. 14, 731-739. doi: 10.1016/j.soard.2018.02.024

Sinal, C. J., Tohkin, M., Miyata, M., Ward, J. M., Lambert, G., and Gonzalez, F. J. (2000). Targeted disruption of the nuclear receptor FXR/BAR impairs bile acid and lipid homeostasis. Cell 102, 731-744. doi: 10.1016/S0092-8674(00)00062-3

Sips, F. L. P., Eggink, H. M., Hilbers, P. A. J., Soeters, M. R., Groen, A. K., and van Riel, N. A. W. (2018). Analysis identifies intestinal transit as a key determinant of systemic bile acid metabolism. Front. Physiol. 9:631. doi: 10.3389/fphys.2018. 00631

Steinert, R. E., Peterli, R., Keller, S., Meyer-Gerspach, A. C., Drewe, J., Peters, T., et al. (2013). Bile acids and gut peptide secretion after bariatric surgery: a 1year prospective randomized pilot trial. Obesity 21, E660-E668. doi: 10.1002/ oby. 20522

Tacchino, R. M., Mancini, A., Perrelli, M., Bianchi, A., Giampietro, A., Milardi, D., et al. (2003). Body composition and energy expenditure: relationship and changes in obese subjects before and after biliopancreatic diversion. Metabolism 52, 552-558. doi: 10.1053/meta.2003.50109

Thomas, C., Gioiello, A., Noriega, L., Strehle, A., Oury, J., Rizzo, G., et al. (2009). TGR5-mediated bile acid sensing controls glucose homeostasis. Cell Metab. 10, 167-177. doi: 10.1016/j.cmet.2009.08.001

Tilg, H., and Kaser, A. (2011). Gut microbiome, obesity, and metabolic dysfunction. J. Clin. Investig. 121, 2126-2132. doi: 10.1172/JCI58109

Trabelsi, M. S., Daoudi, M., Prawitt, J., Ducastel, S., Touche, V., Sayin, S. I., et al. (2015). Farnesoid X receptor inhibits glucagon-like peptide-1 production by enteroendocrine L cells. Nat. Commun. 6:7629. doi: 10.1038/ncomms8629

Tremaroli, V., Karlsson, F., Werling, M., Stahlman, M., Kovatcheva-Datchary, P., Olbers, T., et al. (2015). Roux-en-Y gastric bypass and vertical banded gastroplasty induce long-term changes on the human gut microbiome contributing to fat mass regulation. Cell Metab. 22, 228-238. doi: 10.1016/j. cmet.2015.07.009

Turnbaugh, P. J., Hamady, M., Yatsunenko, T., Cantarel, B. L., Duncan, A., Ley, R. E., et al. (2009). A core gut microbiome in obese and lean twins. Nature 457 480-484. doi: 10.1038/nature07540

Turnbaugh, P. J., Ley, R. E., Hamady, M., Fraser-Liggett, C. M., Knight, R., and Gordon, J. I. (2007). The human microbiome project. Nature 449, 804-810. doi: $10.1038 /$ nature 06244
Turnbaugh, P. J., Ley, R. E., Mahowald, M. A., Magrini, V., Mardis, E. R., and Gordon, J. I. (2006). An obesity-associated gut microbiome with increased capacity for energy harvest. Nature 444, 1027-1031. doi: 10.1038/nature 05414

Vrieze, A., Van Nood, E., Holleman, F., Salojarvi, J., Kootte, R. S., Bartelsman, J. F., et al. (2012). Transfer of intestinal microbiota from lean donors increases insulin sensitivity in individuals with metabolic syndrome. Gastroenterology 143, 913-6e7. doi: 10.1053/j.gastro.2012.06.031

Wang, H., Chen, J., Hollister, K., Sowers, L. C., and Forman, B. M. (1999). Endogenous bile acids are ligands for the nuclear receptor FXR/BAR. Mol. Cell 3, 543-553. doi: 10.1016/S1097-2765(00)80348-2

Watanabe, M., Houten, S. M., Mataki, C., Christoffolete, M. A., Kim, B. W., Sato, H., et al. (2006). Bile acids induce energy expenditure by promoting intracellular thyroid hormone activation. Nature 439, 484-489. doi: 10.1038/ nature 04330

Watanabe, M., Houten, S. M., Wang, L., Moschetta, A., Mangelsdorf, D. J., Heyman, R. A., et al. (2004). Bile acids lower triglyceride levels via a pathway involving FXR, SHP, and SREBP-1c. J. Clin. Invest. 113, 1408-1418. doi: 10.1172/JCI21025

Wu, T., Bound, M. J., Standfield, S. D., Gedulin, B., Jones, K. L., Horowitz, M. et al. (2013). Effects of rectal administration of taurocholic acid on glucagon-like peptide- 1 and peptide YY secretion in healthy humans. Diabetes Obes. Metab. 15, 474-477. doi: 10.1111/dom.12043

Wu, T., Ma, J., Bound, M. J., Checklin, H., Deacon, C. F., Jones, K. L., et al. (2014). Effects of sitagliptin on glycemia, incretin hormones, and antropyloroduodenal motility in response to intraduodenal glucose infusion in healthy lean and obese humans and patients with type 2 diabetes treated with or without metformin. Diabetes 63, 2776-2787. doi: 10.2337/db131627

Wu, T., Rayner, C. K., and Horowitz, M. (2016). Incretins. Handb. Exp. Pharmacol. 233, 137-171. doi: 10.1007/164_2015 9

Zhang, H., DiBaise, J. K., Zuccolo, A., Kudrna, D., Braidotti, M., Yu, Y., et al. (2009). Human gut microbiota in obesity and after gastric bypass. Proc. Natl. Acad. Sci. U.S.A. 106, 2365-2370. doi: 10.1073/pnas.0812600106

Zhang, X., Liu, T., Wang, Y., Zhong, M., Zhang, G., Liu, S., et al. (2016). Comparative effects of bile diversion and duodenal-jejunal bypass on glucose and lipid metabolism in male diabetic rats. Obes. Surg. 26, 1565-1575. doi: 10.1007/s11695-015-1925-y

Zhang, X., Young, R. L., Bound, M., Hu, S., Jones, K. L., Horowitz, M., et al. (2019). Comparative effects of proximal and distal small intestinal glucose exposure on glycemia, incretin hormone secretion, and the incretin effect in health and type 2 diabetes. Diabetes Care 42, 520-528. doi: 10.2337/dc18-2156

Zhang, Y., Lee, F. Y., Barrera, G., Lee, H., Vales, C., Gonzalez, F. J., et al. (2006). Activation of the nuclear receptor FXR improves hyperglycemia and hyperlipidemia in diabetic mice. Proc. Natl. Acad. Sci. U.S.A. 103, 1006-1011. doi: 10.1073/pnas.0506982103

Zhernakova, A., Kurilshikov, A., Bonder, M. J., Tigchelaar, E. F., Schirmer, M., Vatanen, T., et al. (2016). Population-based metagenomics analysis reveals markers for gut microbiome composition and diversity. Science 352, 565-569. doi: $10.1126 /$ science.aad3369

Conflict of Interest Statement: The authors declare that the research was conducted in the absence of any commercial or financial relationships that could be construed as a potential conflict of interest.

The handling Editor declared a past supervisory role with one of the authors XZ.

Copyright (C) 2019 Wang, Cheng, Wang, Dai, Zhang and Hu. This is an open-access article distributed under the terms of the Creative Commons Attribution License (CC BY). The use, distribution or reproduction in other forums is permitted, provided the original author(s) and the copyright owner(s) are credited and that the original publication in this journal is cited, in accordance with accepted academic practice. No use, distribution or reproduction is permitted which does not comply with these terms. 